\title{
Solution Behavior of Surfactants in Ethylene Glycol: Probing the Existence of a CMC and of Micellar Aggregates
}

\author{
R. NAgarajan ${ }^{1}$ And Chien-Chung Wang \\ Department of Chemical Engineering, The Pennsylvania State University, 161 Fenske Laboratory, University Park, Pennsylvania 16802
}

Received March 21, 1995; accepted September 11, 1995

\begin{abstract}
We explore the solution behavior of surfactants in ethylene glycol from a theoretical point of view utilizing the thermodynamic approach developed earlier for aqueous solutions. In this approach, the standard free energy change on aggregation has three solvent-dependent contributions, namely, (a) the surfactant tail transfer free energy, (b) the aggregate core- solvent interfacial free energy, and (c) the free energy of ionic headgroup interactions at the aggregate surface. We develop estimates for these free energy contributions corresponding to ethylene glycol as the solvent and use them to compute the size distribution of aggregates for alkyl $\left(C_{12}, C_{14}, C_{16}\right)$ pyridinium bromides and alkyl $\left(C_{10}, C_{12}, C_{14}, C_{16}\right)$ trimethyl ammonium bromides. For all surfactants considered, the theory predicts that aggregates form only at very large surfactant concentrations in contrast to the behavior exhibited by these surfactants in water. Further, the predicted aggregation numbers are quite small. The aggregates seldom exceed in size small oligomers when the surfactant tail length is not too large (for $n_{c}=12$ or below). As a result of the small aggregation numbers, the sizedependent solution properties are shown to change only gradually as a function of the total surfactant concentration rather than exhibiting any sharp transitions, thus contributing to the ambiguity in the precise determination of the critical micelle concentration (C MC ). Finally, we show that the weak cooperativity in the association process, the resulting small sizes for the aggregates and the corresponding uncertainty in the determination of the CMC are all linked mainly to the surfactant tail transfer free energy and the aggregate core- solvent interfacial free energy contributions and not to the ionic headgroup repulsions. 1996 Academic Press, Inc.
\end{abstract}

\section{INTRODUCTION}

Surfactant self-assembly in aqueous solutions has been widely investigated both experimentally and theoretically (1-5). Experimental measurements of properties such as the electrical conductivity, surface tension, dye solubilization capacity, osmotic pressure, and light scattering intensity as a function of the total surfactant concentration show a sharp

\footnotetext{
${ }^{1}$ To whom correspondence should be addressed. E-mail: rxn@psuvm.psu.edu.
}

transition in the value of the measured property over a very narrow range of surfactant concentration. This surfactant concentration is identified as the critical micelle concentration (CMC). At surfactant concentrations below the CMC, mainly singly dispersed molecules and possibly some small aggregates such as dimers and trimers are present in the solution. In contrast, at concentrations above the CMC, micelles containing a large number of surfactant molecules are formed in the solution. At relatively low or moderate surfactant concentrations, these aggregates can assume a variety of shapes such as spherical micelles, slightly asymmetrical globular or ellipsoidal micelles, large rodlike micelles which may be rigid or flexible, and spherical bilayer vesicles. At larger concentrations of surfactants in solution, liquid crystalline aggregates can come into existence.

Significantly different behavior is observed when the surfactant molecules are dissolved in nonpolar solvents $(6,7)$. In such solutions, for many surfactants, the solution properties change only gradually as a function of the surfactant concentration, thus making the experimental identification of a CMC ambiguous, if not impossible. Further, the aggregates formed are usually found to be small, not much larger than oligomers, with aggregation numbers seldom exceeding 10 $\sim 20$. Exceptions to these general observations have been reported in the literature with respect to both the appearance of a sharply defined CMC as well as the formation of large aggregates in nonpolar solvents (8).

The contrasting self-assembly behaviors of surfactants in water and nonpolar solvents has stimulated many studies on the role of solvent properties with respect to aggregation. An obvious approach has involved the replacement of water with other organic solvents characterized by a range of polarities, which allows the surfactant tails to experience a range of solvophobicities. A number of organic solvents including formamide, ethylene glycol, N,N-dimethyl formamide, glycerol, dimethyl sulfoxide, dimethyl acetamide, and N-methyl acetamide, have been examined with respect to the ability of surfactants to aggregate in these solvents $(9,10)$. Conflicting results have appeared in the literature, calling into question whether or not the experimental observations point to the 
existence of a CMC and the formation of micelles. The first claim regarding the existence of micellar aggregates in a nonaqueous polar solvent was made by Ray (10a) on the basis of surface tension measurements made on dodecyl pyridinium bromide $\left(\mathrm{C}_{12} \mathrm{PBr}\right)$ and tetradecyl trimethyl ammonium bromide $\left(\mathrm{C}_{14} \mathrm{TAB}\right)$ in ethylene glycol solutions. From a change in the slope of surface tension curves, CMC values were estimated to be 0.55 and $0.25 \mathrm{M}$, respectively, in these two systems. A calculation of the surface excess concentrations in these two systems yielded values significantly smaller than those found in aqueous solutions. These observations led the author to conclude that micellar aggregates are formed but probably of smaller aggregation numbers. Binana-Limbele and Zana (10d) measured the conductivity of cetyl trimethyl ammonium chloride (CTAC) in ethylene glycol solutions in the concentration range from 0 to $0.6 \mathrm{M}$. They found that in each of the three different ranges of surfactant concentrations where the conductance was plotted against the concentration, small changes in the slopes can be detected, thus allowing the identification of an operational $\mathrm{CMC}$ in each concentration range. More interestingly, they observed a similar change in slope in solutions containing tetramethyl ammonium chloride, a nonamphiphilic organic ion. On this basis, they concluded that no micellar aggregates are formed in CTAC and only some progressive association leading to small aggregates occurs at increasing surfactant concentrations. Sjöberg et al. (10f) employed surface tension and NMR measurements to examine the aggregation of cetyl trimethyl ammonium bromide $\left(\mathrm{C}_{16} \mathrm{TAB}\right)$ in formamide, ethylene glycol, and their mixtures with water. They observed a reasonably sharp break point in the surface tension vs concentration, which they identified as the CMC. The estimated micellar aggregation numbers were smaller by a factor of 0.27 compared to that in water for ethylene glycol and a factor of 0.33 for formamide. Larger aggregates were observed in the binary aqueous-organic mixed solvents. Backlund et al. (10j) explored the aggregation and phase behavior of tetradecyl trimethyl ammonium bromide $\left(\mathrm{C}_{14} \mathrm{TAB}\right)$ in ethylene glycol. They concluded on the basis of conductivity and density measurements that the identification of a CMC was impossible. The differential conductivity plot, in surfactant concentration range 0.16 to $0.36 \mathrm{M}$, failed to reveal any feature characteristic of the presence of aggregates. They did observe aggregation of $\mathrm{C}_{14} \mathrm{TAB}$ in the form of liquid crystals over a narrow concentration range in ethylene glycol. Gharibi et al. (10e, 10g) employed emf measurements with surfactant selective membrane electrodes to determine cmc values for alkyl pyridinium bromides $\left(\mathrm{C}_{n} \mathrm{PBr}\right)$ and alkyl trimethyl ammonium bromides $\left(\mathrm{C}_{n} \mathrm{TAB}\right)$. No measurements of aggregation numbers were made. At concentrations below the CMC, they interpreted the nonideality revealed in the emf measurements in terms of the formation of dimers and trimers.
Although many experimental measurements have been reported in the literature, no attempt has been made to theoretically predict the aggregation behavior of surfactants in these polar, organic solvents. The main goal of this paper is to apply the theory of self-assembly in aqueous solutions, developed earlier by us (4b), to the study of self-assembly in polar organic solvents in order to predict a priori properties such as the CMC, micelle size distribution, and aggregate polydispersity in these solvents. Also, the free energy model is used to elucidate the differences between the aggregation behavior in water and that in polar organic solvents. In this paper, the model predictions have been obtained for ethylene glycol as the solvent since it has been widely studied experimentally (10). Ethylene glycol is a nonaqueous protic solvent which has been widely used in protein conformation studies and to simulate membranes. It is known that both inter- and intramolecular hydrogen bonds are formed in ethylene glycol, though they are less strong than those in water. For illustrative purposes, predictive calculations have been performed for alkyl trimethyl ammonium bromides $\left(\mathrm{C}_{n} \mathrm{TAB}\right)$ and alkyl pyridinium bromides $\left(\mathrm{C}_{n} \mathrm{PBr}\right)$, for which experimental $\mathrm{CMC}$ values have been reported in the literature $(10 \mathrm{e}, 10 \mathrm{~g})$.

\section{THERMODYNAMICS OF MICELLIZATION}

The basic thermodynamic relations that describe the aggregation behavior of surfactants are well known in the literature (1-5) and only a brief presentation is included here. For a surfactant solution containing solvent molecules, singly dispersed surfactant molecules, and aggregates of various aggregation numbers, $g$, the equilibrium condition of a minimum in the Gibbs free energy stipulates

$$
\mu_{g}^{0}+k T \ln X_{g}=g\left(\mu_{1}^{0}+k T \ln X_{1}\right) \text {, }
$$

where $X_{1}$ and $X_{g}$ are the mole fractions of the singly dispersed molecules and aggregates of size $g$, respectively, and $\mu_{1}^{0}$ and $\mu_{g}^{0}$ are their respective standard chemical potentials, defined as those corresponding to infinitely dilute solution conditions. From Eq. [1], one can calculate the size distribution of the aggregates and using this size distribution, all sizedependent solution properties can be estimated.

In order to calculate $X_{g}$ based on Eq. [1], we need an explicit expression for $\left(\mu_{g}^{0}-g \mu_{1}^{0}\right)=g \Delta \mu_{g}^{0}$ as a function of the size and shape of the micelles. Here, $\Delta \mu_{g}^{0}$ is the difference in the standard chemical potential between a surfactant molecule in an aggregate of size $g$ and a singly dispersed surfactant molecule in the solvent. From a purely geometrical point of view, aggregates of small aggregation numbers can pack as spheres while larger aggregates pack into globular or ellipsoidal shapes. Further, very large aggregates having rod-like shapes can also form in surfactant 
solutions. However, the aggregates formed in the systems explored in this paper are found to be small, and hence only the equations appropriate for the spherical geometry of the micelles are presented here. For a spherical micelle of aggregation number $g$, we denote the micelle core radius by $R$ while $a$ refers to the surface area per molecule of the micellar core. If the hydrophobic tail of the surfactant has a volume $v_{\mathrm{s}}$ and extended length $l_{\mathrm{s}}$, the micelle geometry is specified by

$$
V_{g}=g v_{\mathrm{s}}=\frac{4 \pi R^{3}}{3}, \quad A_{g}=g a=\frac{4 \pi R^{2}}{g}, \quad l_{\mathrm{s}} \leqslant R,
$$

where $V_{g}$ is the volume and $A_{g}$ is the surface area of the micelle core. For hydrocarbon surfactants, the molecular volume $v_{\mathrm{s}}$ of the tail consisting of $n_{\mathrm{c}}$ carbon atoms is calculated from the group contributions of $\left(n_{\mathrm{c}}-1\right)$ methylene groups and the terminal methyl group. At temperature $T$ (in $\mathrm{K}$ ),

$$
\begin{aligned}
& v\left(\mathrm{CH}_{3}\right)=54.6+0.124(T-298) \AA^{3}, \\
& v\left(\mathrm{CH}_{2}\right)=26.9+0.0146(T-298) \AA^{3} .
\end{aligned}
$$

The extended length $l_{\mathrm{s}}$ of the surfactant tail is calculated from the group contribution of $1.265 \AA$ for the methylene group and $2.765 \AA$ for the terminal methyl group.

In writing Eq. [2], we have assumed that no solvent can penetrate the core region of the aggregate. While this is an excellent assumption when water is the solvent, it may not hold equally well for nonaqueous solvents which are characterized by better mutual solubility with hydrocarbons compared to water. The present treatment, however, applies to the limiting case of no solvent penetration in the aggregate core and the possibility of solvent presence in the core and its implications remain to be explored.

\section{FREE ENERGY MODEL AND ESTIMATION OF MODEL PARAMETERS}

Expressions for various free energy contributions to $\Delta \mu_{g}^{0}$ have been formulated in our earlier work (4b) by considering the changes in the intermolecular interactions accompanying the aggregation process. Specifically, these contributions account for the following factors: (a) the surfactant tail is removed from contact with the solvent and is transferred to the hydrophobic core of the micelle $\left(\Delta \mu_{g}^{0}\right)_{\mathrm{tr}}$, (b) the surfactant tail inside the micelle has a conformation different from that in a pure hydrocarbon liquid because of packing constraints imposed inside the micelle $\left(\Delta \mu_{g}^{0}\right)_{\text {def }},(\mathrm{c})$ the formation of the micelle creates an interface between the hydrophobic micellar core and the solvent $\left(\Delta \mu_{g}^{0}\right)_{\text {int }},(\mathrm{d})$ the polar headgroups of the surfactants at the micelle surface exhibit steric repulsions $\left(\Delta \mu_{g}^{0}\right)_{\text {ste }}$, and (e) the polar head- groups, if they are ionic, also exhibit at the micelle surface mutual electrostatic repulsions $\left(\Delta \mu_{g}^{0}\right)_{\text {ionic }}$. Expressions to compute the above free energy contributions, adapted for ethylene glycol as the solvent, are briefly presented below. For a detailed discussion of the origin of these expressions, along with the parameter values valid for aqueous solutions, Ref. (4b) may be consulted.

\section{Transfer Free Energy of the Surfactant Tail}

This contribution is estimated from the infinite dilution activity coefficient data for paraffinic hydrocarbons in ethylene glycol (11). The infinite dilution activity coefficient data are correlated in Ref. (11) to the hydrocarbon chain length at four temperatures in the range between 25 and $90^{\circ} \mathrm{C}$. From these, we develop the following relations for the transfer free energy contributions as a function of temperature. For the methylene $\left(\mathrm{CH}_{2}\right)$ group,

$$
\frac{\left(\Delta \mu_{g}^{0}\right)_{\mathrm{tr}}}{k T}=0.307 \ln T-\frac{428}{T}-0.003083 T,
$$

while for the methyl $\left(\mathrm{CH}_{3}\right)$ group,

$$
\frac{\left(\Delta \mu_{g}^{0}\right)_{\mathrm{tr}}}{k T}=0.149 \ln T-\frac{720}{T}-0.0021 T .
$$

The transfer free energy contribution is negative and is solely responsible for any aggregation to occur. Since this contribution does not depend on the aggregate size $g$, it plays no role in determining the equilibrium structure of the micelles formed.

\section{Deformation Free Energy of the Surfactant Tail}

The surfactant tail inside the micelle is not in a conformational state identical to that of alkyl chains in liquid hydrocarbons. This is because one end of the surfactant tail is constrained to remain at the micelle-solvent interface, while the entire tail has to assume a conformation consistent with the maintenance of uniform density within the micellar core. The consequent deformation of the tail (necessitated by these molecular packing requirements) has been modeled in our earlier work using a lattice treatment and leads to a free energy contribution given by

$$
\frac{\left(\Delta \mu_{g}^{0}\right)_{\mathrm{def}}}{k T}=\left(\frac{9 P \pi^{2}}{80}\right)\left(\frac{R^{2}}{N L^{2}}\right)
$$

where $L$ is the linear dimension of a lattice site taken equal to $4.6 \AA, N$ is the number of lattice sites occupied by the surfactant tail taken to be $l_{\mathrm{s}} / L$, and $P$ is the molecular packing parameter defined as 


$$
P=\frac{V_{g}}{A_{g} R}=\frac{v_{\mathrm{s}}}{a R}
$$

For spherical aggregates, $P=\frac{1}{3}$, in view of Eq. [2]. This is a positive free energy contribution whose magnitude increases with increasing aggregation number. Therefore, this contribution plays a role in restricting the growth of aggregates.

\section{Aggregate Core-Solvent Interfacial Free Energy}

The formation of the micelle generates an interface between the hydrophobic micellar core and the solvent. This free energy contribution is calculated from

$$
\frac{\left(\Delta \mu_{g}^{0}\right)_{\mathrm{int}}}{k T}=\left(\frac{\sigma_{\mathrm{agg}}}{k T}\right)\left(a-a_{0}\right),
$$

where $\sigma_{\text {agg }}$ is the macroscopic hydrocarbon-ethylene glycol interfacial tension, $a$ is the area per molecule of the core region defined by Eq. [2], and $a_{0}$ is the area of the core surface shielded by the surfactant headgroup. This free energy contribution is positive and its magnitude decreases with an increase in the aggregation number. Thus, this contribution promotes the growth of the aggregates and is primarily responsible for the cooperativity of aggregation.

The interfacial tensions between ethylene glycol and paraffinic hydrocarbons have been experimentally measured at $20^{\circ} \mathrm{C}$ and are found to lie in the range of 16 to $19 \mathrm{dyn} / \mathrm{cm}$, depending upon the hydrocarbon chain length (12). We use the approach employed in our study of aqueous solutions to calculate the interfacial tension as a function of both the tail length and the temperature. $\sigma_{\mathrm{agg}}$ is calculated from the surface tensions $\sigma_{\mathrm{s}}$ of the surfactant tail and $\sigma_{\mathrm{EG}}$ of ethylene glycol via the relation

$$
\sigma_{\mathrm{agg}}=\sigma_{\mathrm{s}}+\sigma_{\mathrm{EG}}-2 \psi\left(\sigma_{\mathrm{s}} \sigma_{\mathrm{EG}}\right)^{1 / 2},
$$

where $\psi$ is a constant whose value has been estimated by us to be 0.78 , from knowledge (12) of all the tensions appearing in Eq. [9]. The surface tension $\sigma_{\mathrm{s}}$ of a tail of molecular weight $M$ is evaluated at temperature $T$ (in ${ }^{\circ} \mathrm{K}$ ) using $(4 b)$,

$$
\sigma_{\mathrm{s}}=35.0-325 M^{-2 / 3}-0.098(T-298),
$$

while the surface tension of ethylene glycol is calculated (13) using,

$$
\sigma_{\mathrm{EG}}=48.5-0.1(T-298) \text {. }
$$

All surface and interfacial tensions in the above expressions are given in units of dyn $/ \mathrm{cm}$. As in our earlier work on aqueous micellar solutions (4b), the area $a_{0}$ appearing in Eq. [9] is taken equal to $L^{2}$ if the polar headgroup of the surfactant has an effective cross-sectional area $a_{\mathrm{p}}$ larger than $L^{2}$. If $a_{\mathrm{p}}$ is smaller than $L^{2}$, then the polar head does not fully shield the tail cross section and hence, $a_{0}$ is taken equal to $a_{\mathrm{p}}$.

\section{Headgroup Steric Interactions}

The steric repulsions among the headgroups on the micellar surface provides a free energy contribution that is calculated from

$$
\frac{\left(\Delta \mu_{g}^{0}\right)_{\text {ste }}}{k T}=-\ln \left[1-\frac{a_{\mathrm{p}}}{a}\right]
$$

One may observe the functional form borrowed from the van der Waals equation of state. This free energy contribution is present irrespective of whether the headgroups are nonionic, ionic, or zwitterionic. This free energy contribution is positive and it increases in magnitude with an increase in the aggregation number. Consequently, this contribution serves to restrict the growth of aggregates.

\section{Headgroup Ionic Interactions}

The electrostatic interactions among the ionic headgroups on the micelle surface are calculated using the expression

$$
\begin{aligned}
& \frac{\left(\Delta \mu_{g}^{0}\right)_{\text {ionic }}}{k T}=2\left[\ln \left(\frac{S}{2}+\left[1+\left(\frac{S}{2}\right)^{2}\right]^{1 / 2}\right)\right. \\
& -\frac{2}{S}\left(\left[1+\left(\frac{S}{2}\right)^{2}\right]^{1 / 2}-1\right)-\frac{4}{\kappa S(R+\delta)} \\
& \left.\times \ln \left(\frac{1}{2}+\frac{1}{2}\left[1+\left(\frac{S}{2}\right)^{2}\right]^{1 / 2}\right)\right],
\end{aligned}
$$

where

$$
\begin{aligned}
S=\frac{4 \pi e^{2}}{\epsilon \kappa a_{\delta} k T}, \quad \kappa=\left(\frac{8 \pi n_{0} e^{2}}{\epsilon k T}\right)^{1 / 2} & \\
n_{0} & =\frac{\left(C_{1 \text { ion }}+C_{\mathrm{add}}\right) N_{\mathrm{Av}}}{1000} .
\end{aligned}
$$

Here, $e$ is the electronic charge $\left(4.8 \times 10^{-10}\right.$ esu $), \epsilon$ is the dielectric constant of ethylene glycol, $\delta$ is the distance from the hydrophobic core surface to the surface where the center of the counterion is located, $\kappa$ is the reciprocal Debye length, $n_{0}$ is the number of counterions per $\mathrm{cm}^{3}$ of the solution, $C_{1 \text { ion }}$ is the molar concentration of the singly dispersed surfactant 
molecules, $C_{\text {add }}$ is the molar concentration of the uni-univalent salt added to the solution, $N_{\mathrm{Av}}$ is Avogadro's number, and $a_{\delta}$ is the area per molecule of the micelle calculated at the distance $\delta$ from the hydrophobic core surface given by

$$
A_{g \delta}=4 \pi(R+\delta)^{2}=g a_{\delta} .
$$

This free energy contribution is positive and it increases in magnitude with an increase in the aggregation number. Thus, this contribution along with those of headgroup steric interactions and tail deformations contributes to the anticooperativity of aggregation, restricting the growth of aggregates.

The dielectric constant $\epsilon$ of ethylene glycol has been experimentally measured (14) at various temperatures. We find these data are satisfactorily correlated as a function of temperature $T$ (in $\mathrm{K}$ ) by the expression

$$
\epsilon=46.6 \exp [-0.00516(T-273)] .
$$

\section{Molecular Constants for Surfactants}

In order to proceed with the size distribution calculations based on the various free energy contributions listed above, some molecular constants characterizing the surfactants are needed. The surfactants studied include alkyl pyridinium bromides with $\mathrm{C}_{12}, \mathrm{C}_{14}$, and $\mathrm{C}_{16}$ hydrocarbon chains and alkyl trimethyl ammonium bromides with $\mathrm{C}_{10}, \mathrm{C}_{12}, \mathrm{C}_{14}$, and $\mathrm{C}_{16}$ hydrocarbon chains. For the pyridinium bromide headgroup, using molecular structural models, we estimate the effective cross-sectional area of the polar headgroup $a_{\mathrm{p}}$ to be $34 \AA^{2}$. Consequently, $a_{0}$ is taken to be $21 \AA^{2}$. We also estimate that the center of the counterion is located at a distance $\delta=2.2 \AA$ from the hydrophobic core surface. Similarly, for the alkyl trimethyl ammonium bromide headgroups, we estimate that $a_{\mathrm{p}}=54 \AA^{2}, \delta=3.45 \AA$, and $a_{0}=$ $21 \AA^{2}$. The extended chain lengths $l_{\mathrm{s}}$ and volumes $v_{\mathrm{s}}$ for the different surfactant tails are calculated using the expressions presented earlier as functions of the number of carbon atoms in the tails.

\section{Computational Approach}

The free energy expressions detailed above are introduced in Eq. [1], where $X_{1}$ and $g$ are the independent variables and $X_{g}$ is the dependent variable. We calculate the size distribution $X_{g}$ as a function of $g$ for a specified value of the monomer concentration $X_{1}$. The total surfactant concentration $X_{\text {tot }}$ is then calculated to be $\left(X_{1}+\sum g X_{g}\right)$ in mole fraction units. The surfactant concentrations expressed as molar concentrations $C$ and mole fractions $X$ in this paper are interrelated via the conversion relation

$$
C=1000\left(V_{\text {surf }}+\frac{(1-X)}{X} V_{\mathrm{EG}}\right)^{-1},
$$

where $V_{\text {surf }}$ and $V_{\mathrm{EG}}$ are the molar volumes of the surfactant and ethylene glycol, respectively. The molar volume of the surfactant is calculated knowing the polar headgroup volume of approximately $200 \AA^{3}$ for pyridinium bromide and 190 $\AA^{3}$ for trimethyl ammonium bromide and using the estimates for the tail volume given by Eq. [3] as a function of the tail length $n_{\mathrm{c}}$. The molar volume of ethylene glycol used in the calculations is $55.8 \mathrm{~cm}^{3} / \mathrm{mol}$.

From the calculated size distribution data $\left(X_{g}\right.$ versus $g$ ), the true weight-average and the number-average aggregation numbers $\left(g_{\mathrm{w}}\right.$ and $\left.g_{\mathrm{n}}\right)$, the apparent weight-average and the number-average aggregation numbers $\left(g_{\mathrm{w}, \text { app }}\right.$ and $\left.g_{\text {n,app }}\right)$ can be calculated based on the definitions

$$
\begin{gathered}
g_{\mathrm{w}}=\frac{\sum g^{2} X_{g}}{\sum g X_{g}}, \quad g_{\mathrm{n}}=\frac{\sum g X_{g}}{\sum X_{g}} \\
g_{\mathrm{w}, \text { app }}=\frac{X_{1}+\sum g^{2} X_{g}}{X_{1}+\sum g X_{g}}, \quad g_{\text {n,app }}=\frac{X_{1}+\sum g X_{g}}{X_{1}+\sum X_{g}} .
\end{gathered}
$$

The aggregate polydispersity is given by the ratio $\left(g_{\mathrm{w}} / g_{\mathrm{n}}\right)$, which is known as the polydispersity index. Further, the CMC can be calculated in a number of ways as described in the following section. For comparison purposes, we have also computed the aggregation behavior in water for one of the surfactants, cetyl pyridinium bromide $\left(\mathrm{C}_{16} \mathrm{PBr}\right)$. The free energy expressions corresponding to aqueous solutions along with needed molecular constants are discussed in detail in Ref. [4b] and are not reproduced in this paper.

\section{RESULTS AND DISCUSSION}

\section{Aggregate Size Distribution}

To illustrate the main features of the surfactant solution behavior in ethylene glycol, we first discuss in detail the predictions of the model for the surfactant cetyl pyridinium bromide. The calculated size distribution $X_{g}$ of cetyl pyridinium bromide aggregates in ethylene glycol at two different surfactant concentrations are plotted in Fig. 1. For comparison, the calculated size distribution of aggregates in aqueous solution is shown in Fig. 2. The size distribution curves in ethylene glycol correspond to monomer concentrations of $C_{1}$ $=120$ and $140 \mathrm{mM}$, respectively, while the size distribution curves in water correspond to monomer concentrations of $C_{1}=0.54$ and $0.57 \mathrm{mM}$. A number of contrasting features in these two figures deserve to be noted. First, the total surfactant concentrations $C_{\mathrm{tot}}$, at which the size distributions have been calculated, are 1000-fold larger for ethylene glycol solutions compared to aqueous solutions. Second, a clear maximum in the size distribution corresponding to aggregates of a narrow size range is observed in the aqueous solution, even at the very low surfactant concentrations 


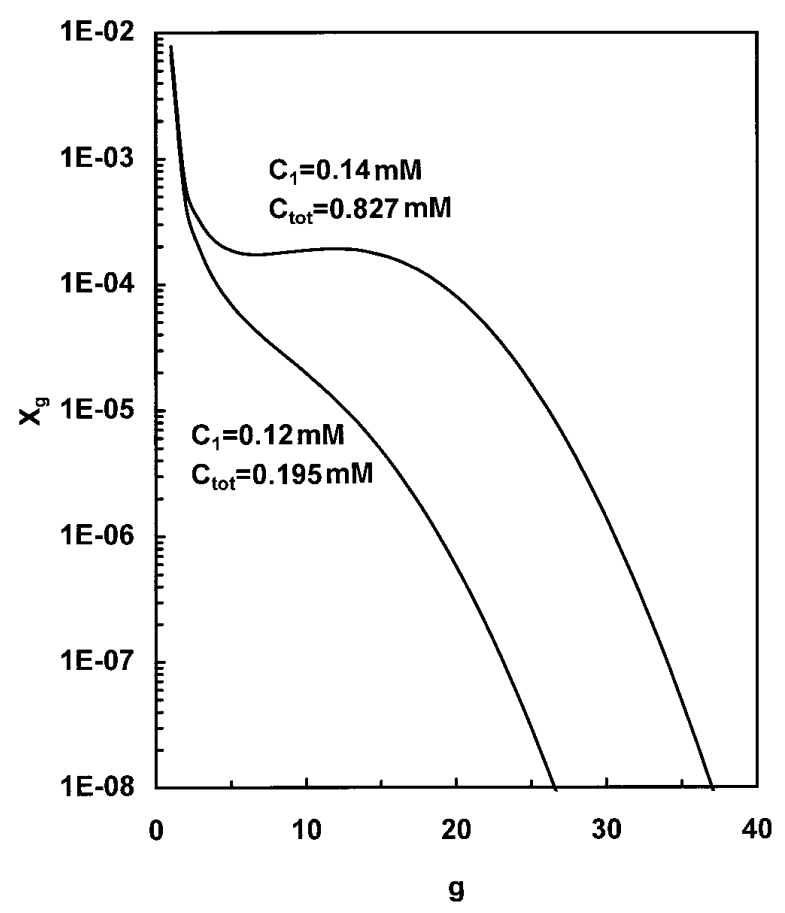

FIG . 1. Calculated size distribution of cetyl pyridinium bromide aggregates in ethylene glycol at two monomer concentrations, $C_{1}=120 \mathrm{mM}$, $\mathrm{C}_{\mathrm{tot}}=195 \mathrm{mM}$ and $C_{1}=140 \mathrm{mM}, C_{\mathrm{tot}}=827 \mathrm{mM}$.

shown in Fig. 2. In contrast, even at the high concentrations shown, the aggregate size distribution in ethylene glycol does not show a pronounced maximum. Third, a change in the monomer concentration by a very small factor of 1.05 causes the aggregate concentration $X_{g}$ to increase by over two orders of magnitude, in the case of aqueous solutions. In contrast, to achieve a similar increase in the aggregate concentration in ethylene glycol solutions, the monomer concentration has to be increased by a factor of 1.17. Finally, the location of a maximum in the aggregate size distribution is not appreciably altered in aqueous solutions when the total surfactant concentration is changed by an order of magnitude as shown in Fig. 2. This is in contrast to the behavior in ethylene glycol (Fig. 1) where an increase in the total surfactant concentration causes aggregates of larger aggregation numbers to become more significant. These differences in the nature of the size distribution curves in water and ethylene glycol give rise to important differences in the nature of the critical micelle concentration and aggregate size in the two solvents, as discussed below.

\section{Critical Micelle Concentration}

The CMC is defined in practice as the surfactant concentration at which the experimentally measured solution property displays a sharp transition in behavior. Because of this operational definition of the CMC, its magnitude depends on the type of experimental measurement being made. For example, surface tension depends largely on the concentration of the monomeric surfactant as the total surfactant concentration is altered. Consequently, a sharp transition in the plot of $X_{1}$ (or $\left.C_{1}\right)$ against the total concentration $X_{\text {tot }}=X_{1}+\sum g X_{g}$ (or $C_{\text {tot }}$ ) can be used to determine the CMC. Properties such as osmotic pressure, vapor pressure, and freezing point depression depend upon the total number of distinct species present in the solution. Therefore, the CMC measured using such techniques can be determined from a plot of the apparent number average aggregation number $g_{\text {n,app }}$ versus the total surfactant concentration. The solubilization of dyes, which is a common technique for determining the $\mathrm{cmc}$, depends upon the concentration of surfactant present in the aggregated form. The CMC measured by the dye solubilization technique can thus be determined by plotting $\sum g X_{g}$ against the total surfactant concentration. In using light scattering to determine the $\mathrm{CMC}$, the scattering intensity is dependent on the average mass of the species in solution. Thus, in this case, the CMC can be determined from a plot of the apparent weight-average aggregation number $g_{\mathrm{w}, \text { app }}$ versus the total surfactant concentration. Besides the approaches discussed above, numerous other methods can be listed for the experimental measurement and/or calculation of the CMC.

The four different ways of determining the CMC discussed above are used to estimate the theoretical CMC of cetyl pyridinium bromide in ethylene glycol (Fig. 3) and in water (Fig. 4),

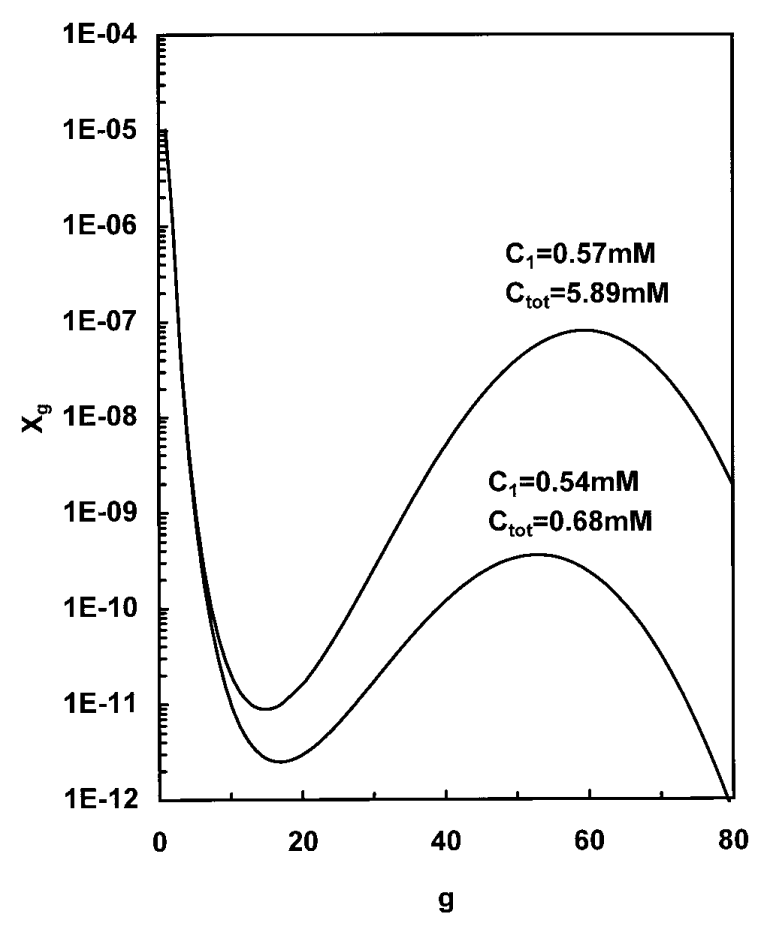

FIG. 2. Calculated size distribution of cetyl pyridinium bromide aggregates in aqueous solution at two monomer concentrations, $C_{1}=0.54 \mathrm{mM}$, $C_{\text {tot }}=0.68 \mathrm{~m} M$ and $C_{1}=0.57 \mathrm{~m} M$ and $C_{\text {tot }}=5.89 \mathrm{mM}$. 


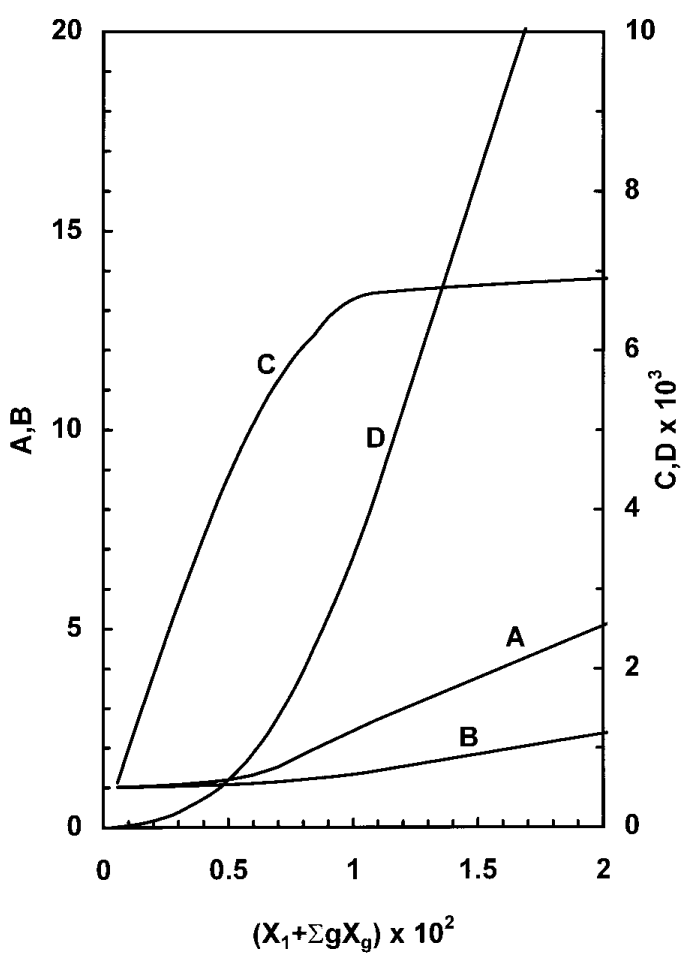

FIG. 3. Calculated size-dependent variables for cetyl pyridinium bromide solutions in ethylene glycol as a function of the total surfactant concentration. A denotes the apparent weight average aggregation number, $\mathrm{B}$ denotes the apparent number average aggregation number, $\mathrm{C}$ denotes the monomer concentration $X_{1}$, and D denotes the total concentration of the surfactant in the form of aggregates, $\Sigma g X_{g}$. All concentrations are expressed in mole fraction units. See text for the definitions of the apparent aggregation numbers.

using the calculated size distribution data. The size-dependent quantities plotted in Fig. 3 for ethylene glycol do not show a very sharp transition as the total surfactant concentration is increased. Although, all the curves reflect the presence of aggregates, the physical properties (represented by the computed variables) change only gradually over a wide range of concentrations. To identify a single concentration in this range as the CMC becomes difficult and there is considerable ambiguity in the determinations made. Table 1 summarizes the CMC values estimated by two of the approaches mentioned above using the curves drawn in Figs. 3 and 4 along with available experimental data. The CMC values determined by the different techniques for aqueous solutions are quite close to one another whereas there is appreciable variation among the estimates of CMC in ethylene glycol obtained by the different approaches. Indeed, different readers looking at the curves in Fig. 3 may arrive at other estimates for the CMCs in ethylene glycol compared to those listed in Table 1.

\section{Aggregate Polydispersity and Concentration-Dependent Growth}

The true weight-average aggregation number $g_{\mathrm{w}}$ and the polydispersity index $\left(g_{\mathrm{w}} / g_{\mathrm{n}}\right)$ calculated as a function of the

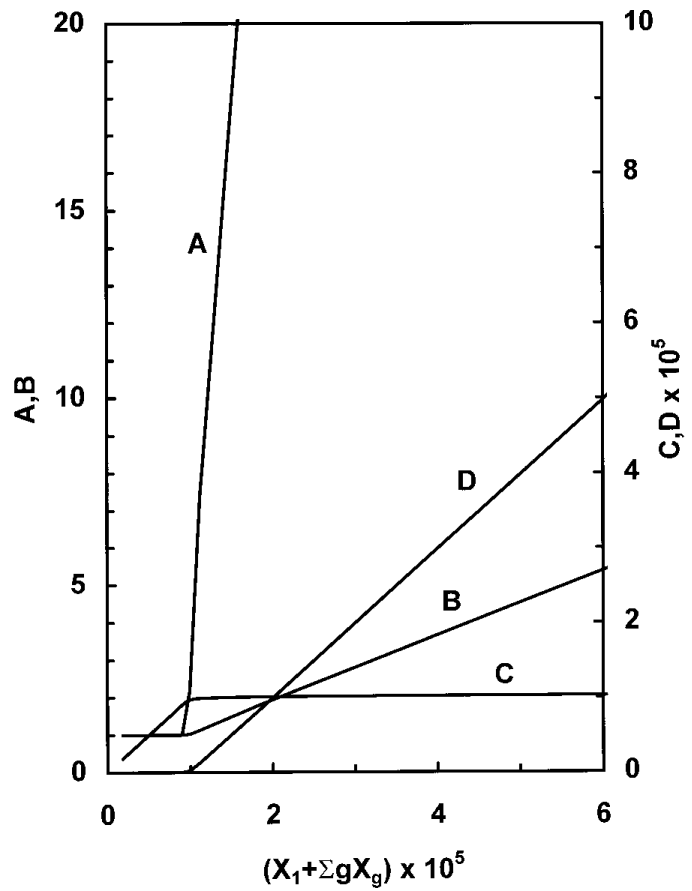

FIG . 4. Calculated size-dependent variables for cetyl pyridinium bromide solutions in water as a function of the total surfactant concentration. The lines denoted by A, B, C, and D refer to the same size-dependent variables as in Fig. 3.

total surfactant concentration are shown in Figs. 5 and 6 for cetyl pyridinium bromide in ethylene glycol. One can see that near the CMC estimated, the solution contains mainly small aggregates such as dimers, trimers, and tetramers. Even at very large surfactant concentrations, the aggregation numbers are very small compared to the typical behavior in water (see Fig. 2, where aggregation numbers of around 60 in aqueous solutions can be observed). The occurrence of dimers and trimers at concentrations below the CMC have

TABLE 1

CMC of Alkyl Pyridinium Bromides and Alkyl Trimethyl Ammonium Bromides in Ethylene $\mathrm{G}$ lycol at $25^{\circ} \mathrm{C}$

\begin{tabular}{lccc}
\hline & $\begin{array}{c}\text { Predicted CMC }(M) \\
\text { based on a plot of } \\
\text { Surfactant }\end{array}$ & $\begin{array}{c}\text { Predicted CMC }(M) \\
\text { based on a plot of } \\
C_{\text {tot }}\end{array}$ & $\begin{array}{c}\text { CMC }(M) \\
\text { based on emf } \\
\text { measurements, } \\
\text { Refs. } \\
(10 \mathrm{e}, 10 \mathrm{~g})\end{array}$ \\
\hline $\mathrm{C}_{16} \mathrm{TAB}$ & 0.112 & 0.095 & 0.10 \\
$\mathrm{C}_{14} \mathrm{TAB}$ & 0.20 & 0.176 & 0.17 \\
$\mathrm{C}_{12} \mathrm{TAB}$ & 0.311 & 0.351 & 0.32 \\
$\mathrm{C}_{10} \mathrm{TAB}$ & 0.60 & 0.646 & 0.44 \\
$\mathrm{C}_{16} \mathrm{PBr}$ & 0.148 & 0.124 & 0.10 \\
$\mathrm{C}_{14} \mathrm{PBr}$ & 0.246 & 0.222 & 0.18 \\
$\mathrm{C}_{12} \mathrm{PBr}$ & 0.446 & 0.372 & 0.65 \\
\hline & & &
\end{tabular}




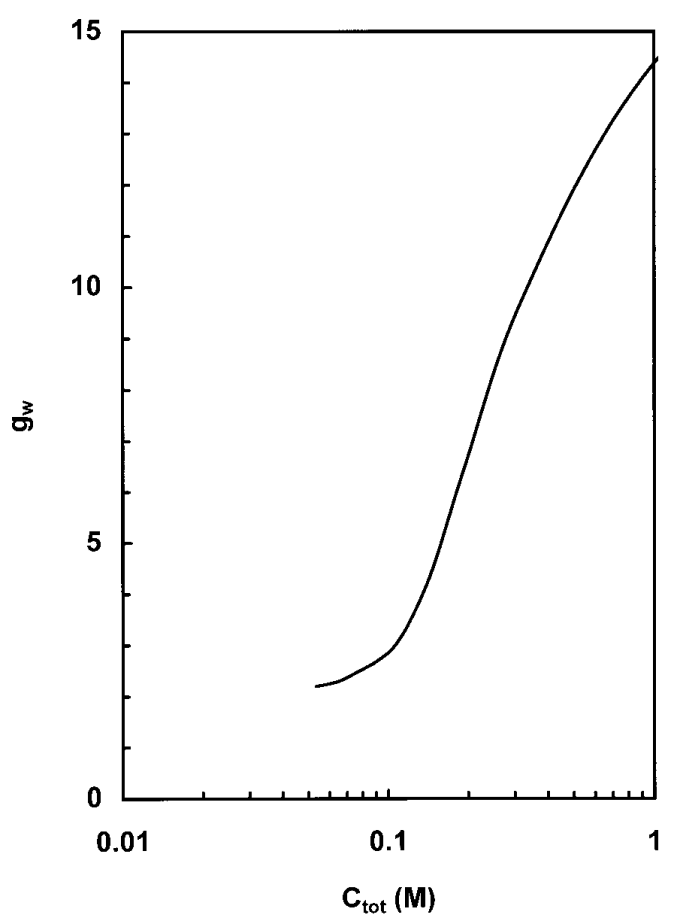

FIG. 5. True weight-average aggregation number of cetyl pyridinium bromide aggregates in ethylene glycol as a function of the total surfactant concentration.

been suggested by Gharibi et al. (10e), based on the interpretation of their emf measurements. The calculated polydispersity index in Fig. 6 shows values appreciably larger than unity, indicating considerable polydispersity in the aggregate sizes. The increasing portion of the curve corresponds to the fact that as the concentration increases, increasing amounts of dimers, trimers, and other smaller oligomers are being formed. Once the aggregates in the size range of about 10 appear in the solution, the polydispersity becomes somewhat reduced and the decreasing portion of the curve is obtained. Over the entire concentration region, the polydispersity values are large. These results may be compared to the polydispersity index of less than 1.05 in aqueous solutions. (One may also note that in aqueous solutions where rodlike micelles form, the polydispersity index is large and close to two. This large value is associated with the linear growth of micellar aggregates. Such a large value, however, is not seen in the ethylene glycol solutions because the aggregates are in the domain of small aggregation numbers only.)

\section{Free Energy Contributions}

The differences observed between aqueous solutions and solutions in ethylene glycol can be quantitatively understood on the basis of the differences in the nature of various free energy contributions to the aggregation process in the two solvents. The calculated free energy curves are shown in
Fig. 7 for ethylene glycol and Fig. 8 for water. The transfer free energy contribution is negative and is responsible for the aggregation. This contribution is independent of the aggregate size and thus has no influence on the size or shape of the equilibrium aggregate formed. All other free energy contributions are positive and are also dependent on the aggregate size. These contributions influence both the magnitude of the CMC as well as the size of the equilibrium aggregate.

The three solvent-dependent free energy contributions show major differences between their values in water and those in ethylene glycol. The first is the surfactant tail transfer free energy which accounts for the solvophobic effect. The magnitude of the transfer free energy is considerably smaller in ethylene glycol compared to that in water. This results in the large magnitude of the CMC in ethylene glycol compared to that in water. The second contribution to note is that of ionic headgroup interactions. The dielectric constant of ethylene glycol is lower than that of water. This should cause an increase in the magnitude of ionic interaction energy in ethylene glycol solutions compared to aqueous solutions. But a comparison of Figs. 7 and 8 show that this is not the case and the ionic interactions in the low dielectric constant medium are smaller, not larger. This is because the monomer concentrations at which aggregates become possible are much larger in ethylene glycol and, conse-

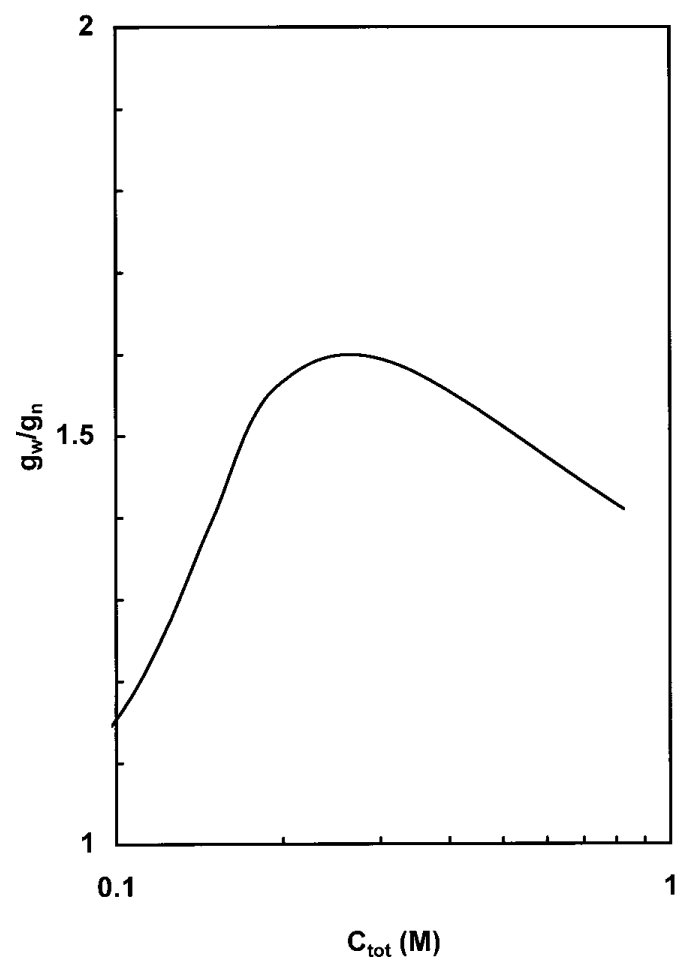

FIG. 6. Polydispersity index of cetyl pyridinium bromide aggregates in ethylene glycol as a function of the total surfactant concentration. 


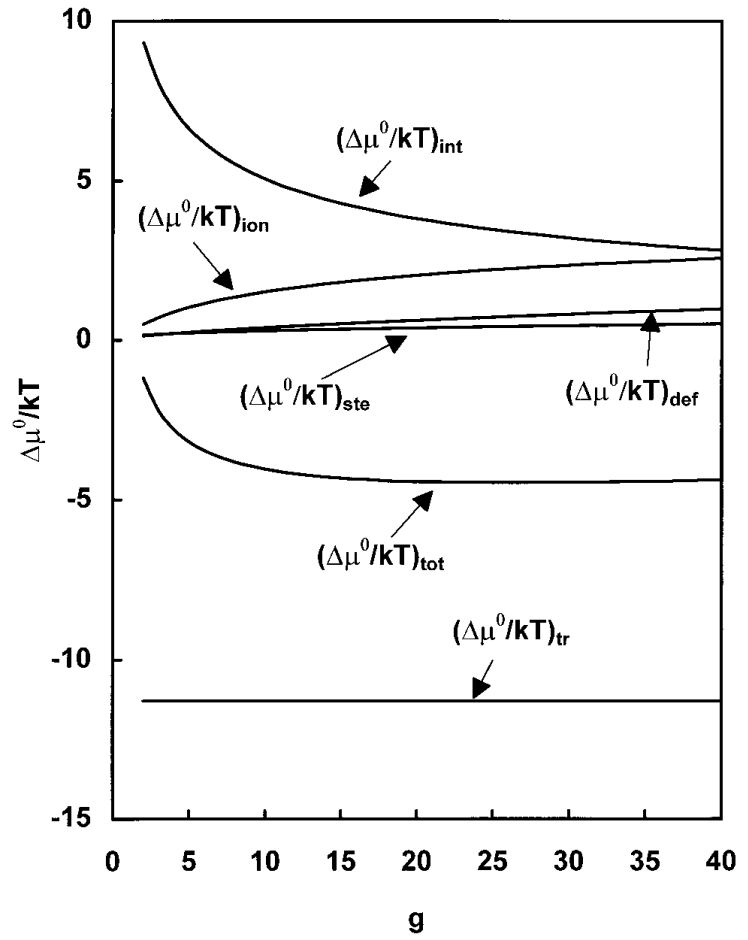

FIG. 7. Contributions to the standard free energy change associated with aggregation of cetyl pyridinium bromide in ethylene glycol as a function of the aggregation number. See text for a discussion of the various contributions.

quently, the ionic strength at the conditions of interest is substantially higher in ethylene glycol solutions compared to in water. The decrease in interionic repulsions due to higher ionic strength more than compensates the increase in ionic repulsions due to the lower dielectric constant of ethylene glycol. The net effect is a decrease in the ionic interaction energies in ethylene glycol solutions compared to that in water. Thus, these interactions contribute to a reduction in the CMC in ethylene glycol and are not responsible for the observed increase in the CMC. Further, one may expect that the decrease in the ionic repulsions will result in larger aggregation numbers for the equilibrium aggregates. But as discussed below, this effect is more than compensated for by the decrease in the interfacial energy which causes the aggregation numbers to become much smaller. The third free energy contribution to note is that of formation of the aggregate core-solvent interface. The interfacial free energy is smaller in the case of ethylene glycol solutions compared to that in water because of the considerably smaller ethylene glycol-hydrocarbon interfacial tension compared to the water-hydrocarbon interfacial tension. This is primarily responsible for the smaller aggregation numbers of the equilibrium aggregates formed in ethylene glycol solutions. Also, the interfacial free energy term causes a reduction in the $\mathrm{CMC}$ in ethylene glycol solutions compared to that in water (because of the lower hydrocarbon-ethylene glycol interfacial tension) and is thus not responsible for the observed increase in the CMC. The steric interaction free energy and the chain deformation free energy are similar in both ethylene glycol and water and are not important in explaining the observed variations in the CMC or in the aggregate sizes.

\section{Aggregation Behavior of Alkyl Pyridinium Bromides}

Computations similar to those discussed in detail above have been carried out for a number of surfactants. Since the general features observed are qualitatively similar to those for cetyl pyridinium bromide, only a few results are presented here. Figure 9 provides a plot of the concentration $C_{1}$ of the singly dispersed surfactant as a function of the total surfactant concentration $C_{\text {tot }}$ for alkyl pyridinium bromides having chain lengths in the range $n_{\mathrm{c}}=12$ to 16 . This plot can be used to estimate the CMC of the surfactants since the variation of the monomer concentration with the total surfactant concentration simulates the surface tension versus concentration behavior, as discussed earlier. The estimated CMC values are shown along with available experimental data $(10 \mathrm{e}, 10 \mathrm{~g})$ in Table 1 . It is evident that the determination of the CMC from this plot is not quite unambiguous as the tail length of the surfactant decreases. Figure 10 presents the calculated apparent weight-average aggregation numbers

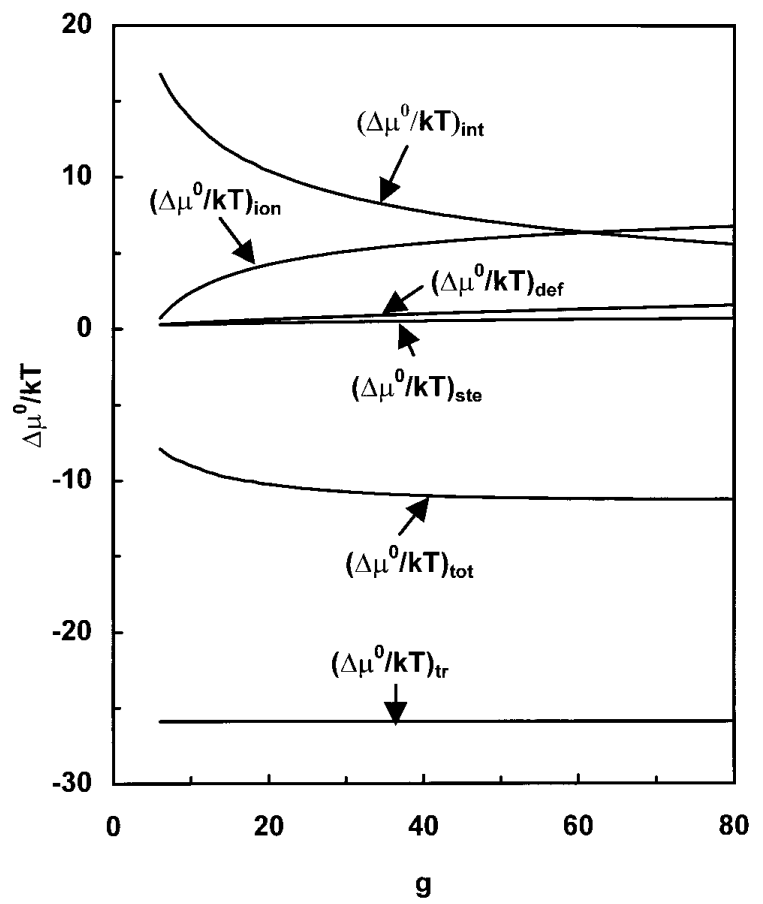

FIG. 8. Contributions to the standard free energy change associated with aggregation of cetyl pyridinium bromide in water as a function of the aggregation number. The various contributions are identical to those described in Fig. 5. 


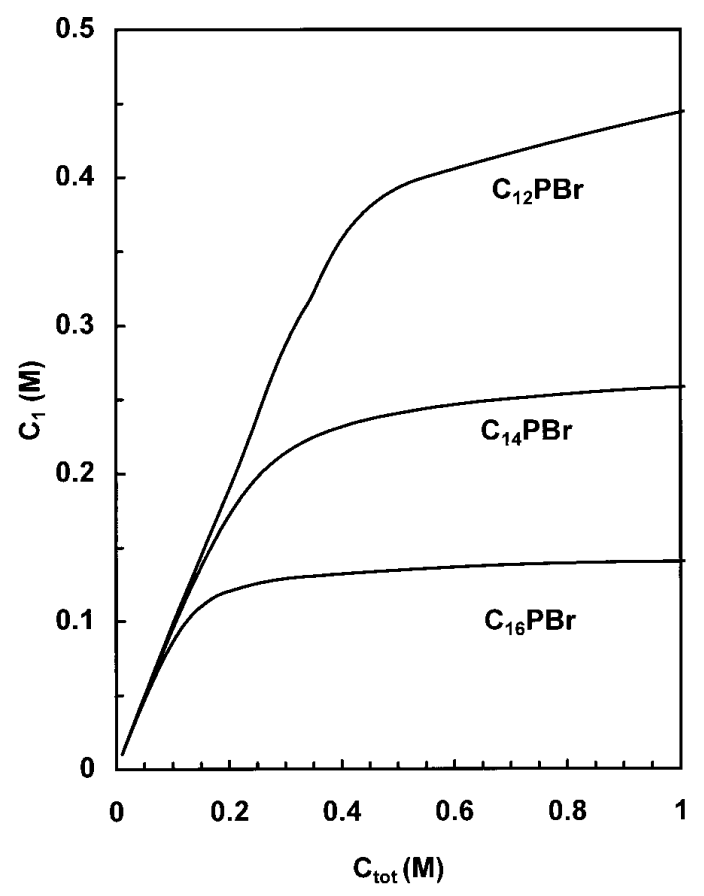

FIG. 9. Calculated dependence of the monomer concentration $C_{1}$ on the total surfactant concentration $C_{\text {tot }}$ for dodecyl, tetradecyl, and cetyl pyridinium bromides in ethylene glycol solutions.

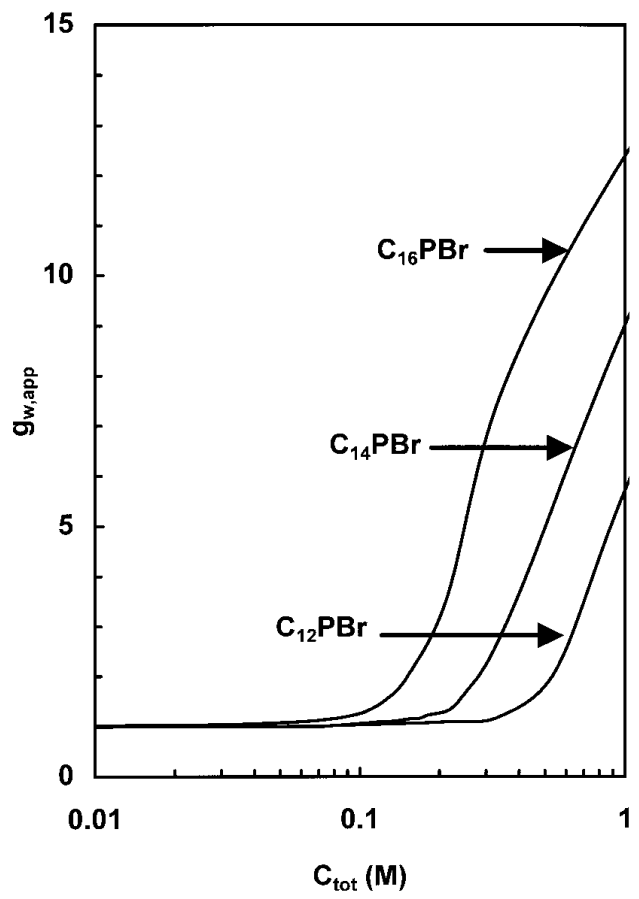

FIG. 10. Calculated dependence of the weight average aggregation number $g_{\mathrm{w}}$ on the total surfactant concentration $C_{\text {tot }}$ for dodecyl, tetradecyl, and cetyl pyridinium bromides in ethylene glycol solutions.

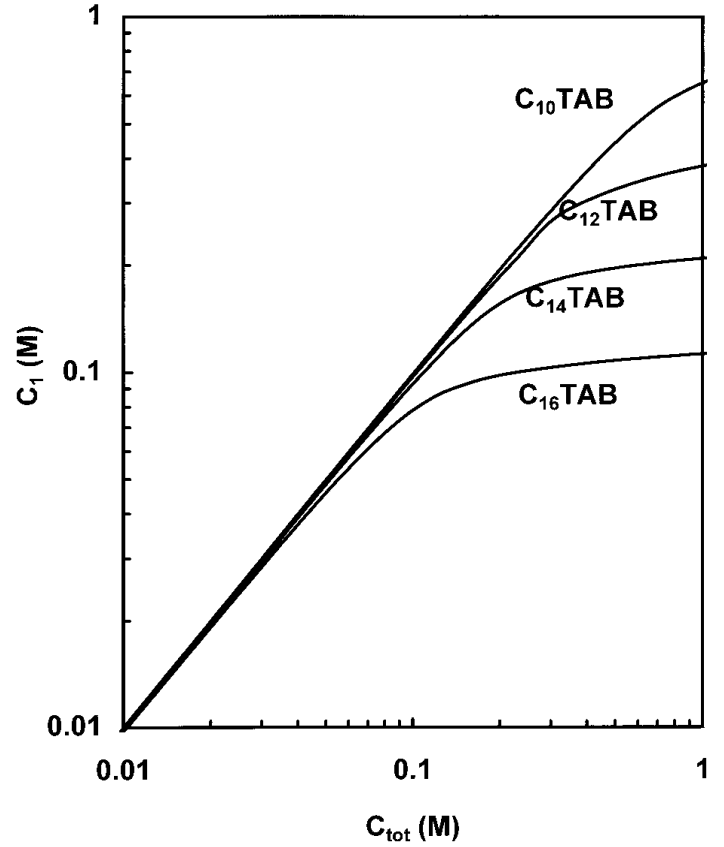

FIG. 11. Calculated dependence of the monomer concentration $C_{1}$ on the total surfactant concentration $C_{\text {tot }}$ for decyl, dodecyl, tetradecyl, and cetyl trimethyl ammonium bromides in ethylene glycol solutions.

as a function of the total surfactant concentration in ethylene glycol solutions. Even at very high concentrations of about $1 \mathrm{M}$, the aggregation numbers are quite small. As one would expect, the aggregation numbers increase with an increase in the tail length of the surfactant.

\section{Aggregation Behavior of Alkyl Trimethyl Ammonium Bromides}

The size distribution of aggregates formed from alkyl trimethyl ammonium bromides having chain lengths in the range $n_{\mathrm{c}}=10$ to 16 have been computed. Figure 11 provides a plot of the concentration $C_{1}$ of the singly dispersed surfactant as a function of the total surfactant concentration $C_{\mathrm{tot}}$. The CMC values estimated using this plot and the available experimental data $(10 \mathrm{e}, 10 \mathrm{~g})$ are also listed in Table 1 . As discussed before, the determination of the CMC from this plot becomes more uncertain as the tail length of the surfactant decreases. Figure 12 presents the calculated apparent weight-average aggregation numbers as a function of the total surfactant concentration in ethylene glycol solutions. Even at very high concentrations of about $1 M$, the aggregation numbers are quite small while the aggregation numbers increase with an increase in the tail length of the surfactant.

\section{Comparison with Experimental Observations in the Literature}

The experimental observations discussed in the literature are explainable using our theoretical predictions. The calcu- 


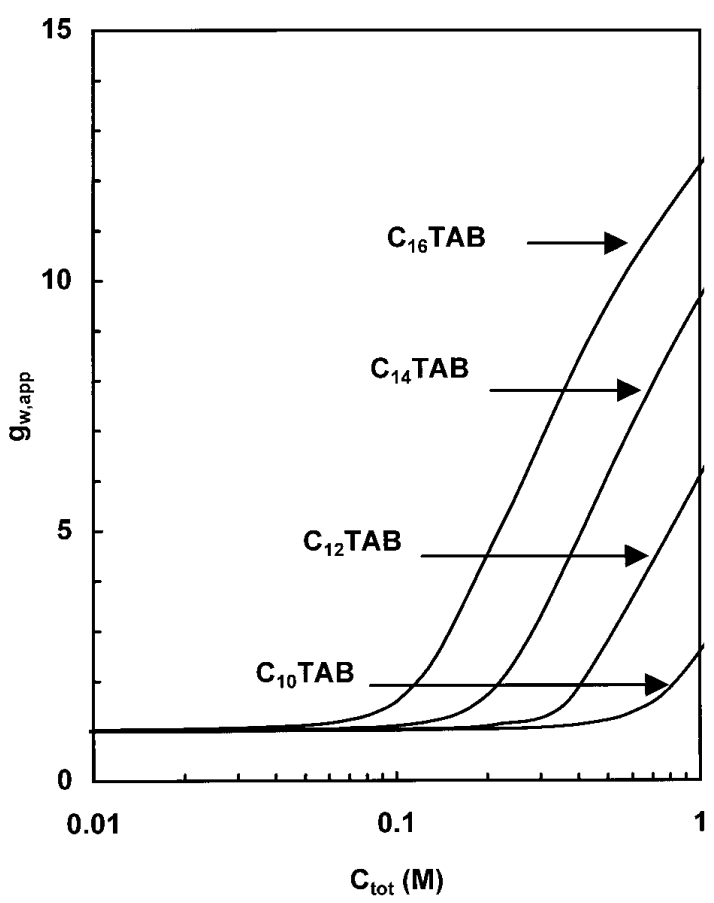

FIG. 12. Calculated dependence of the weight average aggregation number $g_{\mathrm{w}}$ on the total surfactant concentration $C_{\text {tot }}$ for decyl, dodecyl, tetradecyl, and cetyl trimethyl ammonium bromides in ethylene glycol solutions.

lated size-dependent quantities in Fig. 3 show that indeed an operational CMC can be estimated, though not uniquely, based on any one of the many possible experimental techniques. Such a CMC is inherently associated with a larger uncertainty when compared to the usual determinations in aqueous solutions. Further, the model predicts the formation of very small aggregates at equilibrium. For the concentration of $0.36 M \mathrm{C}_{14} \mathrm{TAB}$ (the largest concentration in the differential conductivity plot of Ref. $(10 \mathrm{j})$ ), the predicted apparent weight-average aggregation number is only about 3 (Fig. 12). For the largest concentration of $0.6 \mathrm{M}$ CTAC examined in Ref. (10d), only progressive association with small aggregates was suggested. For $\mathrm{C}_{16} \mathrm{TAB}$ and $\mathrm{C}_{16} \mathrm{PBr}$ (having the same tail length as CTAC but with somewhat different headgroup or counterion), at $0.6 \mathrm{M}$, our model predictions yield an apparent weight average aggregation number of about 10 only (Figs. 10 and 12).

\section{CONCLUSIONS}

The aggregation behavior of surfactants in ethylene glycol has been theoretically investigated by employing the molecular free energy model of aggregation developed earlier for aqueous solutions. Estimates for the solvent-dependent free energy contributions have been obtained accounting for ethylene glycol being the solvent in place of water. Using the model, the size distribution of aggregates, the $\mathrm{CMC}$, and the average aggregation numbers have been calculated. The calculated results show that the operational determination of the CMC values in ethylene glycol has some inherent uncertainty since the size-dependent solution properties change only gradually with increasing surfactant concentration. The magnitudes of the CMC are very much larger in comparison to those observed in aqueous solutions. The large magnitude of the CMC is shown by the model to originate solely from the smaller magnitude of the transfer free energy of the surfactant tail in ethylene glycol solutions. The ambiguity in the determination of the CMC increases as the surfactant tail length decreases and this ambiguity arises from the weak cooperativity of the association process in ethylene glycol. The model also shows that the weak cooperativity and the consequent small aggregation numbers in ethylene glycol arise solely from the smaller magnitude of the interfacial tension in these systems. The ionic interactions at the micellar surface decrease rather than increase despite the lower dielectric constant of ethylene glycol, because of the larger ionic strength associated with the larger monomer concentrations. Thus, this free energy contribution causes only a decrease in the CMC. Also, despite the larger ionic strengths, the aggregation numbers are small because of the dominating influence of the lower interfacial energy. We conclude with the general observation that although an operational CMC may be identified for many surfactants in ethylene glycol, the aggregates are much smaller compared to those in water and can be thought of as small oligomers.

\section{REFERENCES}

1. Tanford, C., "The Hydrophobic Effect." Wiley, New York, 1973.

2. (a) Mukerjee, P., Adv. Colloid Interface Sci. 1, 241 (1967); (b) Mukerjee, P., J. Phys. Chem. 73, 2054 (1969); (c) Mukerjee, P., J. Pharm. Sci. 68, 972 (1974); (d) Mukerjee, P., J. Phys. Chem. 76, 565 (1972).

3. (a) Puvvada, S., and Blankschtein, D., J. Chem. Phys. 92, 3710 (1990); (b) Puvvada, S., and Blankschtein, D., J. Phys. Chem. 96, 5567 (1992); (c) Briganti, G., Puvvada, S., and Blankschtein, D., J. Phys. Chem. 95, 8989 (1991); (d) Carale, T. R., and Blankschtein, D., J. Phys. Chem. 96, 459 (1992).

4. (a) Nagarajan, R., Adv. Colloid Interface Sci. 26, 205 (1986); (b) Nagarajan, R., and Ruckenstein, E., Langmuir 7, 2934 (1991).

5. (a) Israelachvili. J. N., Mitchell, D. J., and Ninham, B. W., J. Chem. Soc. Faraday Trans. 2 72, 1525 (1976); (b) Israelachvili, J. N., Mitchell, D. J., and Ninham, B. W., Biochim. Biophys. Acta 470, 185 (1977); (c) Israelachvili, J. N., Marcelja, S., and Horn, R. G., Q. Rev. Biphys. 13, 121 (1980).

6. Kertes, A. S., and Gutmann, H., in "Surface and Colloid Science", (E. Matijevic, Ed.). Interscience, New York, 1975.

7. Ruckenstein, E., and Nagarajan, R., J. Phys. Chem. 84, 1349 (1980).

8. (a) Kitahara, A., 'Cationic Surfactants.', Dekker, New York, 1970; (b) Fowkes, F. M., in 'Solvent Properties of Surfactants', (K. Shinoda, Ed.). Dekker, New York, 1967.

9. Ward, A. J. I., and du Reau, C., in "Surface and Colloid Science", (E. Matijevic, Ed.), p. 153. Interscience, New York, 1993.

10. (a) Ray, A., J. Am. Chem. Soc. 91, 6511 (1969); (b) Ray, A., and 
Nemethy, G., J. Phys. Chem. 75, 809 (1971); (c) Ionescu, L. G., and Fung, D. S., J. Chem. Soc. Faraday Trans. 1 77, 2907 (1981); (d) Binana-Limbele, W., and Zana, R., Colloid Polym. Sci. 267, 440 (1989); (e) Gharibi, H., Palepu, R., Bloor, D. M., Hall, D. G., and Wyn-Jones, E., Langmuir 8, 782 (1992); (f ) Sjöberg, M., Henriksson, U., and Wärnheim, T., Langmuir 6, 1205 (1990); (g) Palepu, R., Gharibi, H., Bloor, D. M., and Wyn-Jones, E., Langmuir 9, 110 (1993);

(h) Callaghan, A., Doyle, R., Alexander, E., and Palepu, R., Langmuir 9, 3422 (1993); (i) Warnheim, T., and Jonsson, A., J. Colloid Interface
Sci. 125, 627 (1988); (j) Backlund, S., Bergenstahl, B., Molander O., and Warnheim, T., J. Colloid Interface Sci. 131, 393 (1989).

11. Pierotti, G. J., Deal, C. H., and Derr, E. L., Ind. Eng. Chem. 51, 95 (1959).

12. Janczuk, B., Wojcik, W., and Zdziennicka, A., J. Colloid Interface Sci. 157, 384 (1993).

13. Panzer, J., J. Colloid Interface Sci. 44, 142 (1973).

14. Corradini, F., Marcheselli, L., Tassi, L., and Tosi, G., J. Chem. Soc. Faraday Trans. 89, 123 (1993). 